\title{
Particle production at HERA
}

\author{
Anastasia Grebenyuk* \\ DESY, Hamburg, Germany \\ E-mail: Anastasia.Grebenyuk@desy.de
}

\begin{abstract}
Results on particle production in deep-inelastic scattering in $e p$ collision at HERA, obtained with the H1 and ZEUS detectors, are presented. The underlying parton dynamics are investigated by looking at the hardness of the transverse momentum distribution of charged particles and comparing the measurements with Monte Carlo generators based on different approaches for simulating the parton cascade. Studies of the scaled momentum distribution for for $K_{S}^{0}$ and $\Lambda$ particles in the current fragmentation region of the Breit frame in the context of hadronisation are presented. The data are compared to models and to next-to-leading order QCD calculations. In addition, the production of photon at very small angles with respect to the proton beam direction is presented. The cross sections are compared to the predictions of models of deep-inelastic scattering and models of the hadronic interactions of high energy cosmic rays.
\end{abstract}

36th International Conference on High Energy Physics,

July 4-11, 2012

Melbourne, Australia

${ }^{*}$ On behalf of the $\mathrm{H} 1$ and ZEUS collaborations. 


\section{Parton dynamics}

\subsection{Charged particle transverse momentum spectra}

It was suggested in [1] that the transverse momentum spectrum of charged particles is sensitive to whether partons are emitted in a $p_{T}$-ordered cascade (DGLAP) [2] or in an unordered way (beyond DGLAP). The H1 Collaboration has presented measurements [3] of the transverse momenta and pseudorapidities of charged particles and compared them to predictions of various Monte Carlo (MC) generators using different approaches to simulate the parton cascade: the RAPGAP generator [4] based on leading-log DGLAP parton showers; the DJANGOH [5] MC which uses the Colour Dipole Model (CDM) [6] as implemented in ARIADNE [7], which provides a BFKL-like [8, 9] approach; the CASCADE [10] MC generator based on the CCFM model [11]. All generators use the Lund string model [12] for hadronisation as implemented in PythIA [13]. The fragmentation parameter set are tuned by the ALEPH collaboration to fit LEP data [14].

The analysed data set corresponds to an integrated luminosity of $L=88.64 \mathrm{pb}^{-1}$. The analysis is performed in the kinematic phase space region defined by $5<\mathrm{Q}^{2}<100 \mathrm{GeV}^{2}$ and $0.05<y<$ 0.6. The data are corrected for detector effects using MC event samples, as well as for the charged decay products of $K_{S}^{0}, \Lambda$ and for other weakly decaying particles. The transverse momentum and pseudorapidity distributions are presented in the hadronic centre-of-mass system (HCM), i.e. in the rest frame of the virtual photon and proton, and are labeled as $p_{T}^{*}$ and $\eta^{* 1}$, respectively. All distributions shown are normalised to the total number of DIS events in the analysed phase space.

The normalised pseudorapidity distributions for $p_{T}^{*}<1 \mathrm{GeV}$ and for $p_{T}^{*}>1 \mathrm{GeV}$, separately, are shown in Fig. 1. The data are compared to the RAPGAP, DJANGOH and CASCADE generators. As argued in [1], hadronisation effects should be relevant at small $p_{T}^{*}$, while hard parton radiation should manifest itself in the tail of the $p_{T}^{*}$ distribution. To check the sensitivity to hadronisation effects, RAPGAP predictions with default PYTHIA fragmentation parameters and with parameters tuned by ALEPH are shown in Fig. 1. Significant differences between these two models are seen in the soft $p_{T}^{*}$ region, while for particles with $p_{T}^{*}>1 \mathrm{GeV}$ this difference is much smaller. Predictions from generators with different approaches for QCD radiation are also shown in Fig. 1. The best description of the data is achieved by DJANGOH. At large $p_{T}^{*}$ RAPGAP predicts a spectrum which
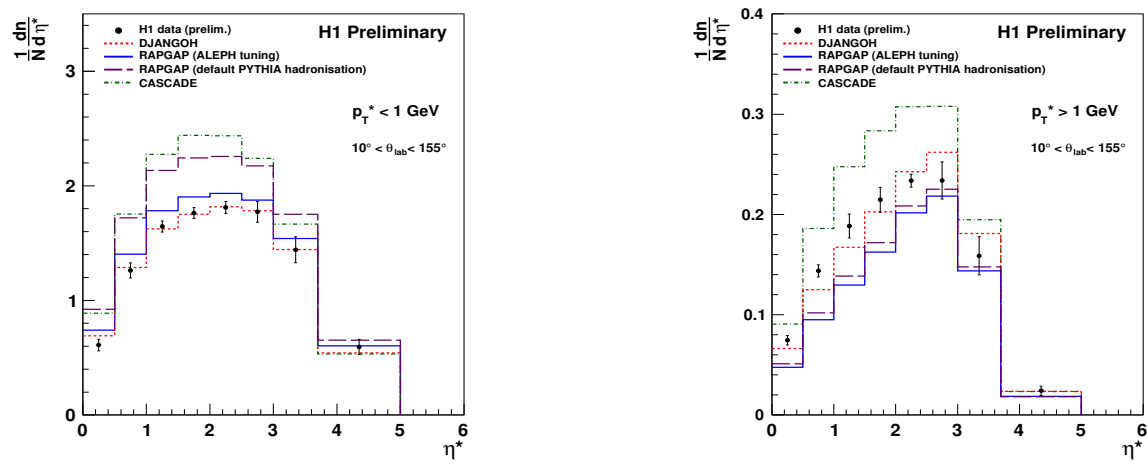

Figure 1: Measured $\eta^{*}$ spectra in the HCM system for the charged particles with $p_{T}^{*}<1 \mathrm{GeV}$ (left) and with $p_{T}^{*}>1 \mathrm{GeV}$ (right) together with RAPGAP, DJANGOH and CASCADE Monte Carlo predictions.

\footnotetext{
${ }^{1}$ The definition of pseudorapidity in the HCM frame is $\eta^{*}=-\ln \left(\tan \left(\theta^{*} / 2\right)\right)$, where $\theta^{*}$ is the angle with respect to the virtual photon direction, i.e. in the positive $z^{*}$ direction.
} 
is too soft towards small $\eta^{*}$. CASCADE predicts too high multiplicities away from the photon direction.

The normalised transverse momentum spectra of charged particles in the pseudorapidity interval of $0<\eta^{*}<1.5$ and in eight different $x$ and $\mathrm{Q}^{2}$ bins are presented in Fig. 2. In the range

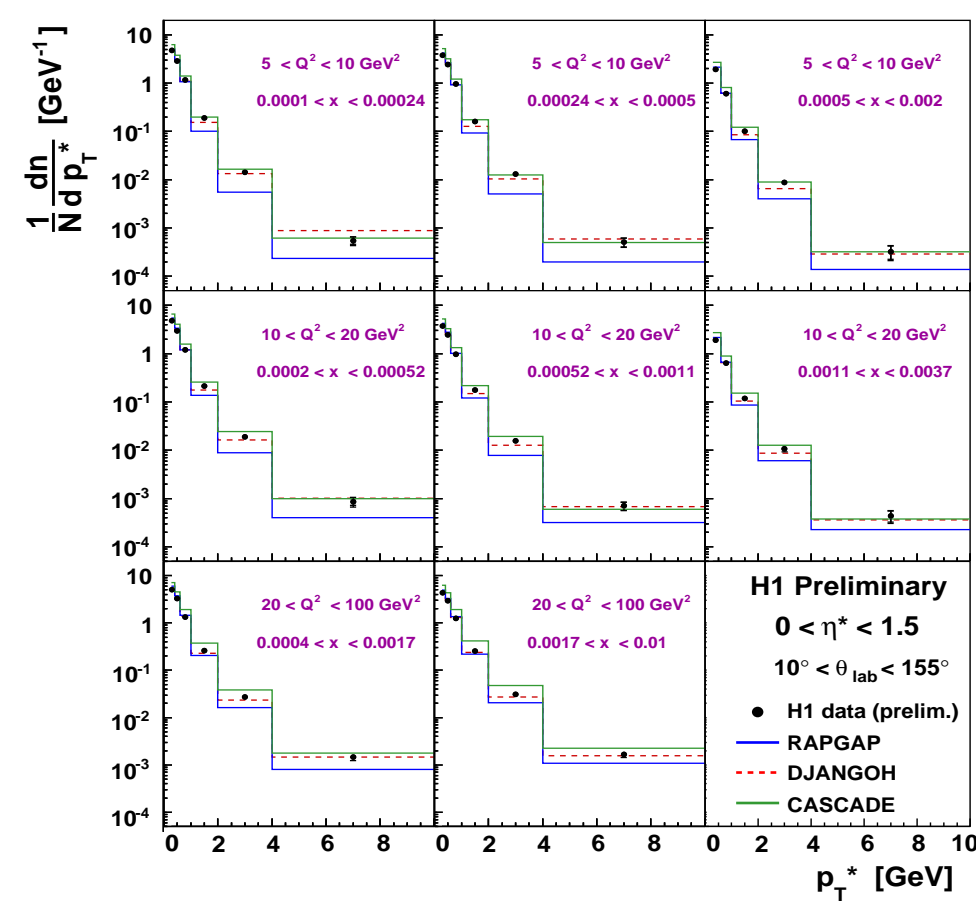

Figure 2: Measured $p_{T}^{*}$ spectra of charged particles in the hadronic centre-of-mass system (HCM) in $0<$ $\eta^{*}<1.5$ range for eight intervals of $\mathrm{Q}^{2}$ and $x_{\mathrm{Bj}}$ together with RAPGAP, DJANGOH and CASCADE Monte Carlo predictions.

$0<\eta^{*}<1.5$ the sensitivity to the parton shower may be studied, while for higher $\eta^{*}$ values the sensitivity to the hard scatter is mainly expected. The CDM model provides a good description of the data over the full kinematic range. The RAPGAP strongly undershoots the data in the lowest $x$ and $\mathrm{Q}^{2}$ bin. The description becomes better at larger values of $x$ and $\mathrm{Q}^{2}$. CASCADE describes the data in the lowest $x$ and $\mathrm{Q}^{2}$ bin and high $p_{T}^{*}$ but has a much harder spectrum at high $x$ and $Q^{2}$.

\section{Hadronisation}

\subsection{1 $K_{S}^{0}$ and $\Lambda$ scaled momentum distributions}

An investigation of the production of $K_{S}^{0}$ mesons and $\Lambda$ baryons in the current fragmentation region of the Breit frame is presented. Multiplicity distributions are measured as a function of $\mathrm{Q}^{2}$ per unit of the scaled momentum, $x_{p}=2 p^{\text {Breit }} / \mathrm{Q}$. Here, $p^{\text {Breit }}$ denotes the momentum of a hadron in the Breit frame. The aim is to check the universality of the quark fragmentation function and the factorisation theorem approach used to predict hadron production in different processes.

The data presented in this section are corrected for detector acceptance, efficiency and resolution effects. All distributions shown are normalised to the total number of DIS events, $N$, in the analysed phase space. Scaled momentum distributions for $K_{S}^{0}$ mesons and $\Lambda$ baryons were mea- 
sured by ZEUS [15] in the kinematic range $10<\mathrm{Q}^{2}<40000 \mathrm{GeV}^{2}$ and $0.001<x<0.75$. The data correspond to an integrated luminosity of $L=290 \mathrm{pb}^{-1}$.

Figure 3 shows the scaled momentum spectra, as a function of $\mathrm{Q}^{2}$ in different regions of $x_{p}$, for $K_{S}^{0}$ (left) and $\Lambda$ (right) production. Large scaling violations are seen: with increasing $\mathrm{Q}^{2}$ the phase

ZEUS

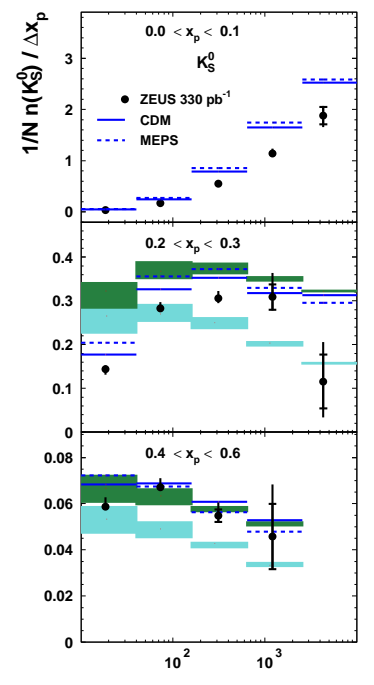

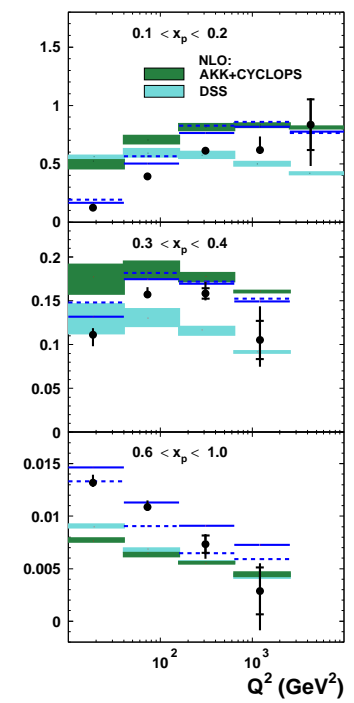

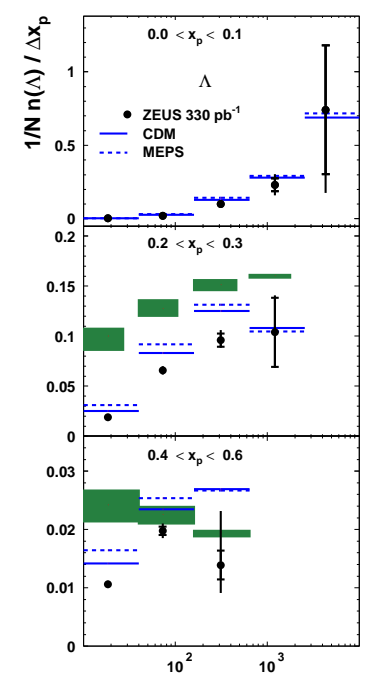

ZEUS

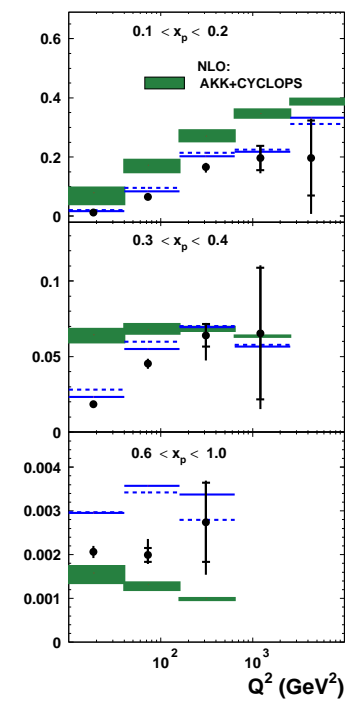

Figure 3: Scaled momentum distributions as a function of $\mathrm{Q}^{2}$ in different regions of $x_{p}$ for $K_{S}^{0}$ (left) and $\Lambda$ (right) and together with predictions of NLO QCD and LO Monte Carlo programs.

space for soft gluon radiation gets enlarged, which leads to a rise in the number of soft particles at small $x_{p}$ and to a decrease in the number of those with high $x_{p}$.

The data are compared to two sets of the NLO QCD predictions based on different parametrisation of the fragmentation functions. The first set was obtained from fits to $e^{+} e^{-}$data and based on the program CYCLOPS [16], called "AKK+CYCLOPS" [17]. The second set was obtained from a global fit to $e^{+} e^{-}, p p$ and $e p$ data, called "DSS" [18]. Both NLO QCD predictions describe the data only in certain regions of the phase space. For the AKK+CYCLOPS calculation this is limited to $0.6<x_{p}<1$, whereas the DSS calculation describes the $K_{S}^{0}$ data adequately, except for regions of low $x_{p}$ and $Q^{2}$. The scaling violation in both NLO calculations is less pronounced than in the data. Together with the NLO QCD calculations the predictions of MC generators ARIADNE based on CDM and the MEPS model of LEPTO [19], based on DGLAP parton shower, are shown in Fig. 3. Both predictions give a reasonable description of the data.

\subsubsection{Production of very forward photon in DIS}

Measurements of particle production at very small angles with respect to the proton beam direction (forward direction) in positron-proton collisions are important for the understanding the fragmentation of the proton remnant. These measurements also provide important constraints for the modeling of the high energy air showers.

In this analysis [20] photons are detected at very small angles, $\eta>7.9$. It relies on the upgraded H1 Forward Neutron Calorimeter (FNC) [21]. The FNC consist of the Main Calorimeter and the Preshower Calorimeter. Events are restricted to the kinematic range $6<\mathrm{Q}^{2}<100 \mathrm{GeV}^{2}$ and 
$0.05<y<0.6$. Forward photons are required to have a signal only in the Preshower calorimeter, and to have $\eta>7.9$ and an energy above $92 \mathrm{GeV}$, which corresponds to the longitudinal momentum fraction $x_{L}=E_{\gamma} / E_{p}>0.1$, where $E_{p}$ and $E_{\gamma}$ are the proton beam and forward photon energy, respectively. The data are corrected for the detector effects and QED radiation.

The normalised differential cross sections for the most energetic forward photons (leading photon) are presented in Fig. 4 as a function of $x_{L}^{\text {lead }}$. The data are compared to the predictions of the MC generators ARIADNE based on CDM and LEPTO as well as the predictions of several hadronic interaction models which are commonly used for the simulation of cosmic ray air shower cascades: EPOS [22], QGSJET 01 [23], QGSJET II-03 [24] and SIBYLL [25]. In all of these models, the main source of forward photons is the decay of $\pi^{0}$ mesons produced from the hadronisation of the proton remnant. The ratios of MC model predictions to the measurements are shown separately. All models overestimate the total rate of forward photons. The best description is achieved by
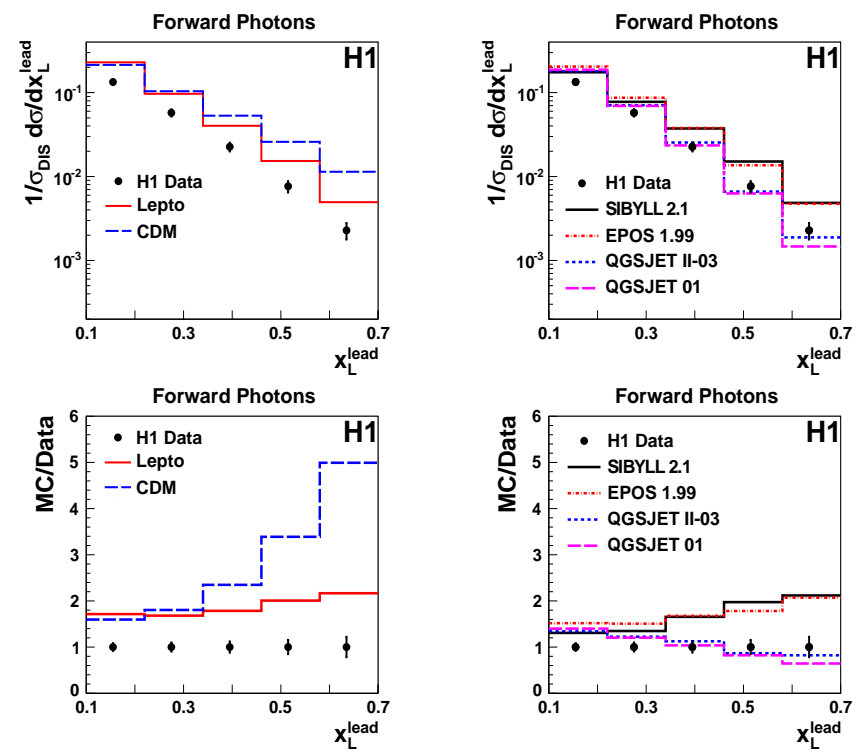

Figure 4: Normalised cross sections of forward photon production as a function of $x_{L}^{\text {lead }}$. The data are compared to two predictions of the DJANGOH Monte Carlo simulation, using LEPTO and CDM to simulate higher orders. The lower row shows the ratios of the Monte Carlo predictions to the data.

the QGSJETs models, which are consistent with the data at high $x_{L}^{\text {lead }}$ values, but in general have steeper behavior than data. The shapes of all measured distributions are well described by LEPTO.

The measurement of forward photons allows a test of the limiting fragmentation hypothesis, according to which the production of forward photons in DIS is insensitive to $\mathrm{Q}^{2}$ and $x_{\mathrm{Bj}}$. The ratio of the forward photon production cross section to the inclusive DIS cross section is measured as a function of $\mathrm{Q}^{2}$ and $x_{\mathrm{Bj}}$, as shown in Fig. 5. The fraction of DIS events with forward photons is independent from $\mathrm{Q}^{2}$ and $x_{\mathrm{Bj}}$ in agreement with the limiting fragmentation hypothesis.

\section{References}

[1] M. Kuhlen, Phys. Lett. B382 (1996) 441.

[2] V. Gribov and V. Lipatov, Sov. J. Nucl. Phys. 15 (1972) and 675;

V. Lipatov, Sov. J. Nucl. Phys. 20 (1975) 94;

G. Altarelli and G. Parisi, Nucl. Phys. B126 (1977) 298; 

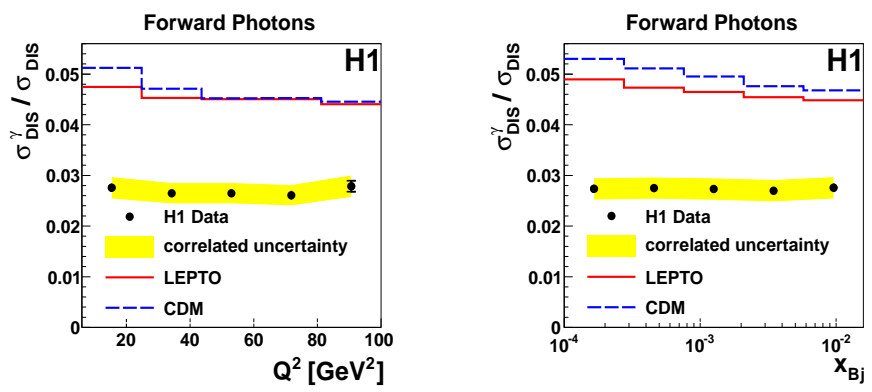

Figure 5: Fraction of DIS events with forward photons as a function of $\mathrm{Q}^{2}$ and $x_{\mathrm{Bj}}$. The expectation from the LEPTO and CDM models are also shown.

Y. Dokshitzer, Sov. Phys. JETP 46 (1977) 641.

[3] H1 Preliminary DIS2011, H1prelim-11-035.

[4] H. Jung, Comp. Phys. Commun. 86 (1995) 147.

[5] K. Charchula, G.A. Schuler and H. Spiesberger, Comp. Phys. Commun. 81 (1994) 381.

[6] B. Andersson et al., Z. Phys. C43 (1989) 625;

L. Lönnblad, Comput. Phys. Commun. 71 (1992) 15.

[7] L. Lönnblad, ARIADNE 4.10, Comput. Phys. Commun. 71 (1992) 15.

[8] E. Kuraev, V. Lipatov and V. Fadin, Sov. Phys. JETP 44 (1976) 443, Sov. Phys. JETP 45 (1977) 199; Y. Balitsky and V. Lipatov, Sov. J. Nucl. Phys. 28 (1978) 822.

[9] L. Lönnblad, Z.Phys. C65, (1995) 285;

A.H. Mueller, Nucl. Phys. B415, (1994) 373.

[10] H. Jung, Comp. Phys. Commun. 143 (2002) 100.

[11] M. Ciafaloni, Nucl. Phys. 296 (1988) 49;

M. Catani, F. Fiorani and G. Marchesini, Phys. Lett. 234 (1990) 339, Nucl. Phys. 336 (1990) 18.

[12] B. Andersson, G. Gustafson, G. Ingelman and T. Sjostrand, Phys. Rept. 97 (1983) 31.

[13] T. Sjostrand et al., Pythia6.1, Comp. Phys. Commun. 135 (2001) 238.

[14] S. Schael et al. [ALEPH Collaboration], Phys. Lett. B606 (2005) 265;

G. Rudolph [ALEPH Collaboration], private communication.

[15] H. Abramowicz et al. [ZEUS Collaboration], JHEP 03 (2012) 020 [arXiv:1111.3526].

[16] D. Graudenz, Phys. Rev. B406 (1997) 178.

[17] S. Albino et al., Phys. Rev. D75 (2007) 034018;

S. Albino, B.A. Kniehl and G. Kramer, Nucl. Phys. B803 (2008) 42.

[18] D. de Florian, R. Sassot and M. Stratmann, Phys. Rev. D75 (2007) 114010.

[19] G. Ingelman et al., Comput. Phys. Commun. 101 (1997) 108.

[20] F.D. Aaron et al. [H1 Collaboration], Eur.Phys.J. C71 (2011) 1771.

[21] F.D. Aaron et al. [H1 Collaboration], Eur. Phys. J. C68 (2010) 381 [arXiv:1001.0532].

[22] K. Werner, F.M. Liu and T. Pierog, Phys. Rev. C74 (2006) 044902 [hep-ph/0506232].

[23] N.N. Kalmykov and S.S. Ostapchenko, Phys. Atom. Nucl. 56 (1993) 346;

N.N. Kalmykov, S.S. Ostapchenko and A.I. Pavlov, Nucl. Phys. Proc. Suppl. 52 B (1997) 17.

[24] S.S. Ostapchenko, Phys. Rev. D74 (2006) 014026 [hep-ph/0505259];

S.S. Ostapchenko, AIP Conf. Proc. 928 (2007) 118 [arXiv:0706.3784].

[25] J. Engel et al., Phys. Rev. D46 (1992) 5013;

E.J. Ahn et al., Phys. Rev. D80 (2009) 094003 [arXiv:0906.4113]. 